This article is licensed under the Creative Commons Attribution-NonCommercial 4.0 International License (CC BY-NC) (http://www.karger.com/Services/OpenAccessLicense). Usage and distribution for commercial purposes requires written permission.

\title{
Fungal Endogenous Endophthalmitis Secondary to Magnusiomyces capitatus
}

\author{
Nestore Rota ${ }^{a} \quad$ Carla Danese $^{a} \quad$ Francesca Menchini $^{\mathrm{a}} \quad$ Silvia Pignatto $^{\mathrm{a}}$ \\ Maddalena Peghin ${ }^{c}$ Matteo Bassettic Paolo Lanzetta ${ }^{a}$ b \\ ${ }^{\text {aD }}$ epartment of Medicine - Ophthalmology, University of Udine, Udine, Italy; \\ bIstituto Europeo di Microchirurgia Oculare - IEMO, Udine, Italy; ${ }^{\mathrm{C} D e p a r t m e n t}$ of \\ Medicine - Infectious Diseases, University of Udine, Udine, Italy
}

\section{Keywords}

Fungal endogenous endophthalmitis · Magnusiomyces capitatus · Geotrichum capitatum

\begin{abstract}
We report the case of a 68-year-old immunocompetent patient with a dilatation of the ascending aorta, intraluminal vegetations, and pseudoaneurysmatic bulging who presented with unilateral fungal endogenous endophthalmitis 8 days after coronary angiogram. The isolated pathogen resulted to be Magnusiomyces capitatus, a filamentous, yeast-like fungus that can be commonly found in normal human microflora, with an immunosuppression-related pathogenicity. A literature research revealed a single case of ophthalmic infection - a keratitis caused by this pathogen. Furthermore, we add a review of mycotic endophthalmitis related to aortic infection.

(C) 2019 The Author(s)

Published by S. Karger AG, Basel
\end{abstract}

\section{Introduction}

Fungal endogenous endophthalmitis is a severe ocular disease associated with potentially poor visual outcomes, derived from hematogenous propagation of a mycotic agent into the eye. Affected patients usually have predisposing systemic conditions, such as immunosuppression (iatrogenic, diabetes mellitus, malignancy, or HIV) indwelling catheters, intravenous 


\section{Case Reports in Ophthalmology}

drug use, or recent major surgical procedures. However, this condition occasionally occurs also in healthy, immunocompetent patients. The most commonly involved pathogen is the yeast Candida albicans, followed by molds such as Aspergillus fumigatus [1]. In detail, almost half of the patients with endophthalmitis caused by Aspergillus species have a condition of iatrogenic immunosuppression and organ transplantation and require a more aggressive therapy, while $C$. albicans-related endophthalmitis has a more indolent course, and patients usually present with a better visual acuity (VA) [2]. Generally, the diagnosis is clinical, and many patients have positive fungal cultures from extraocular sites; therefore, intraocular cultures are not mandatory for each patient. If the diagnosis is unclear, confirmatory laboratory testing, such as cultures or polymerase chain reaction from vitreous tap, can be performed. Treatment is usually represented by systemic antifungals and intravitreal antifungal injections, alone or in combination with pars plana vitrectomy (PPV) [3].

\section{Case Presentation}

We report the case of an immunocompetent 68-year-old patient who was admitted to the emergency department due to angina. He had undergone coronary artery bypass graft operation for obstructive coronaropathy 4 years earlier. Coronary angiogram was urgently performed and revealed an enlargement of the ascending aorta with intraluminal thrombus-like material partially ulcerating the aortic wall, associated with severe stenosis of the coronary arteries. The patient was hospitalized since ascending aorta replacement and multiple coronary arteries bypass graft was indicated. After 8 days, while waiting for surgery, he started complaining of redness and blurred vision in the right eye (RE). The ophthalmological past medical history was unremarkable. VA was 20/125 in the RE and 20/20 in the left eye. On clinical examination of the RE, he presented conjunctival injection, posterior synechiae, Tyndall $2+$, no hypopion, and faint anterior chamber cells $1+$. He was phakic with a moderate cataract (2+) (Fig. 1a). Severe vitritis with 4+ haze was also present. Despite the severe vitreous inflammation and poor pupil dilatation, at fundus biomicroscopy, a rounded, circumscribed, white-yellowish retinal lesion, slightly greater than 1 disc diameter in size, was found in the temporal mid-periphery of the retina (Fig. 1c). At optical coherence tomography (OCT), the lesion appeared as a preretinal, round-shaped hyperreflective lesion with a homogeneous internal pattern, laying on the retinal surface; the retinal layers underneath the lesion were not clearly visible due to a posterior shadowing effect (Fig. 1d). Ultrasound examination showed inflammatory signs in the vitreous chamber, represented by multiple moderately reflective mobile dot echoes, and a preretinal lesion with intermediate echogenicity in the transverse Bscan of the temporal retina (Fig. 1b). Ocular features were clinically consistent with fungal endophthalmitis. The left eye was normal. Broad-spectrum antimycotic therapy with voriconazole, both intravenous ( $6 \mathrm{mg} / \mathrm{kg}$ every $12 \mathrm{~h}$ for $24 \mathrm{~h}$, then $4 \mathrm{mg} / \mathrm{kg}$ every $12 \mathrm{~h}$ for 7 days) and intravitreal $(100 \mu \mathrm{g} / 0.1 \mathrm{~mL})$, was immediately started, with subsequent modest visual improvement to 20/100 in the RE. A vitreous tap was also performed at the time of intravitreal therapy.

After 2 days, the patient became hemodynamically unstable, thus requiring urgent cardiac surgery intervention. What seemed to be an intra-aneurysmal thrombus during coronary angiography revealed to be an intraluminal mycotic vegetation. Microbiological examinations disclosed colonies of Magnusiomyces capitatus, sensible to liposomal amphotericin B. In addition, the vitreous tap resulted positive for the same pathogen. Therefore, endovenous therapy with liposomal amphotericin B was started at the dosage of $3 \mathrm{mg} / \mathrm{kg}$ daily. 


\section{Case Reports in Ophthalmology}

This confirmed the initial clinical diagnosis of endogenous endophthalmitis in the RE, with the source of primary mycotic infection at the level of the aortic wall. Three weeks after cardiac surgery, although no signs of worsening were disclosed, ocular examination did not show any improvement despite the therapy administered. VA in RE was stable at 20/100. Therefore, 30 days after the initial systemic and intravitreal therapy with voriconazole, a more aggressive approach was decided, and the patient underwent 27-gauge three-port PPV and phacoemulsification with intraocular lens implantation associated with an intravitreal injection of amphotericin B ( $5 \mu \mathrm{g} / 0.1 \mathrm{~mL})$ under peribulbar anesthesia. After the initial core vitrectomy, the infective lesion became clearly visible and was removed using both forceps and the vitreous cutter. During surgery, an additional small focus was disclosed at the posterior pole (Fig. 2a). Endolaser was performed over the area where the larger infective focus was located. The RE VA progressively improved up to 20/25 two weeks after ocular surgery. On examination, the anterior and the vitreous chambers were clear, and there was no evidence of recurrence of retinal infiltrate (Fig. 2b-e). Eight months after surgery, VA is maintained at 20/25 without evidence of any signs of inflammation.

\section{Discussion}

We report a case of endogenous endophthalmitis in an immunocompetent patient caused by the pathogen M. capitatus (formerly Geotrichum capitatum), a filamentous yeast-like fungus colonizing human skin and the mucosa of the respiratory and digestive tracts, which produces rapidly proliferating, flat, white colonies with acute-angled hyphae and abundant conidia formation [4].

A literature search revealed a single case of ocular infection caused by this pathogen, a keratomycosis in a patient affected by sarcoidosis who had previously undergone corneal transplantation for a postinfectious corneal scar 5 years before. However, no signs of vitreous inflammation and endophthalmitis were noted. The patient was treated with topical, intracameral and systemic voriconazole, followed by corneal transplantation. The cultures of the aqueous were positive for M. capitatus [5].

An additional search focused on endophthalmitis associated with mycotic aortic aneurysm highlighted 2 cases. The first was a case of Aspergillus endophthalmitis occurring 14 days after percutaneous coronary intervention in a patient affected by pseudoaneurysmatic dilatation of the ascending aorta. The patient presented with reduced VA and vitreous flare and underwent PPV associated with an intravitreal injection of amphotericin B. The authors were not able to provide data on postsurgical visual and anatomic outcomes since the patient had a prolonged course complicated by intracerebral bleeding and respiratory failure and died on postoperative day 39 [6]. The second case was an immunocompetent patient who had undergone bioprosthetic aortic valve replacement three months earlier with a pseudoaneurysm of the ascending and mid-descending aorta caused by Aspergillus, spreading to the eye and causing endophthalmitis. He was treated with systemic antimycotic therapy (amphotericin-B, voriconazole). However, the prognosis was unfavorable, and the patient died after a prolonged hospital course [7].

Since our patient was immunocompetent, we hypothesize that the primary event was a clinically silent mycotic infection of the ascending aorta, mimicking an aneurysm that then spread to the RE, causing endogenous endophthalmitis.

Current recommendations for the treatment of endogenous fungal endophthalmitis are different according to the precise etiology. More specifically, for Candida endophthalmitis, the 
best treatment appears to be PPV associated with intravitreal injection of amphotericin or voriconazole and systemic antifungal therapy, which has to be maintained for either at least 6 weeks or until the resolution of ocular involvement. Immunocompromised patients need the same treatment. However, in the case that they cannot tolerate surgery, intravitreal injection with voriconazole and amphotericin should be performed at baseline and repeated as needed. Voriconazole is also used to treat infections resistant to fluconazole and amphotericin B. It is also indicated against Paecilomyces and Fusarium species. Guidelines for the treatment of Aspergillus endophthalmitis recommend amphotericin $\mathrm{B}$, both intravenously and intravitreally, potentially associated with PPV in sight-threatening cases; as an alternative, either systemic or intravitreal voriconazole may be used [8]. It cannot be excluded that the seeding of the infection was promoted by the insertion of the coronary angiography catheter in the ascending aorta where the mycotic material was located. We decided to treat our patient initially with intravitreal and systemic voriconazole, since we preferred not to perform vitrectomy until hemodynamic stability was reached. After cardiac surgery had been performed, PPV was considered viable. It must be noted that, after obtaining the microbiological results on the infectious material, antifungal therapy was shifted from voriconazole to liposomal amphotericin B, to which the pathogen resulted sensitive.

To our knowledge, this is the first reported case of endogenous endophthalmitis caused by M. capitatus.

The case is unusual not only for the clinical presentation - mycotic endophthalmitis in an immunocompetent patient, caused by an uncommon pathogen - but also for the primary site of infection and the mechanism of fungal spread. Our case and the others described above were all managed with medical and surgical therapy, with favorable visual and anatomical outcomes in our case.

\section{Statement of Ethics}

This work has been performed according to the guidelines for human studies and has been performed ethically in accordance with the World Medical Association Declaration of Helsinki. The subject has given informed consent to publish the case, including publication of images. Information revealing the subject's identity has been avoided. The patient is not identified by the real name.

\section{Disclosure Statement}

Nestore Rota: none;

Carla Danese: none;

Francesca Menchini: Novartis Pharma AG, consultant;

Maddalena Peghin: Pfizer, financial support; MSD, financial support; Dia Sorin, financial support;

Matteo Bassetti: Angelini, financial support, recipient; AstraZeneca, financial support, recipient; Bayer, financial support, recipient; Cidara, financial support, recipient; Cubist, financial support, recipient; Pfizer, financial support, recipient; Menarini, financial support, recipient; MSD, financial support, recipient; Nabriva, financial support, recipient; Paratek, financial support, recipient; Roche, financial support, recipient; Shionogi, financial support, 
recipient; Tetraphase, financial support, recipient; The Medicine Company financial support, recipient; Astellas Pharma Inc., financial support, recipient;

Paolo Lanzetta: Bayer, consultant; Centervue, consultant; Novartis Pharma AG, consultant.

\section{References}

1 Sridhar J, Flynn HW Jr, Kuriyan AE, Miller D, Albini T. Endogenous fungal endophthalmitis: risk factors, clinical features, and treatment outcomes in mold and yeast infections. J Ophthalmic Inflamm Infect. 2013 Sep;3(1):60.

2 Riddell Iv J, McNeil SA, Johnson TM, Bradley SF, Kazanjian PH, Kauffman CA. Endogenous Aspergillus endophthalmitis: report of 3 cases and review of the literature [Internet]. Medicine (Baltimore). 2002 Jul;81(4):311-20. [cited 2019 Jan 4].

3 Relhan N, Schwartz SG, Flynn HW Jr. Endogenous Fungal Endophthalmitis: An increasing problem among intravenous drug users. JAMA. 2017 Aug;318(8):741-742.

4 Arendrup MC, Boekhout T, Akova M, Meis JF, Cornely OA, Lortholary 0; European Society of Clinical Microbiology and Infectious Diseases Fungal Infection Study Group; European Confederation of Medical Mycology. ESCMID and ECMM joint clinical guidelines for the diagnosis and management of rare invasive yeast infections [Internet]. Clin Microbiol Infect. 2014 Apr;20 Suppl 3:76-98.

5 Shah A, Mauger T. Magnusiomyces capitatus: a new and emerging pathogen linked to keratomycosis. Digit J Ophthalmol. 2017 Sep;23(3):75-7.

6 Khumri TM, Joslin NB, Nayyar S, Main ML. Transesophageal echocardiographic diagnosis of Aspergillus fumigatus aortitis after percutaneous coronary intervention. J Am Soc Echocardiogr. 2006 Aug;19(8):1072.e9-11.

7 Rocco JM, Benson MK. Aspergillus aortitis in an immunocompetent patient presenting with acute endophthalmitis. Infect Dis Rep. 2018 Sep;10(2):7750.

8 Sadiq MA, Hassan M, Agarwal A, Sarwar S, Toufeeq S, Soliman MK, et al. Endogenous endophthalmitis: diagnosis, management, and prognosis. J Ophthalmic Inflamm Infect. 2015 Nov;5:32. 


\section{Case Reports in Ophthalmology}
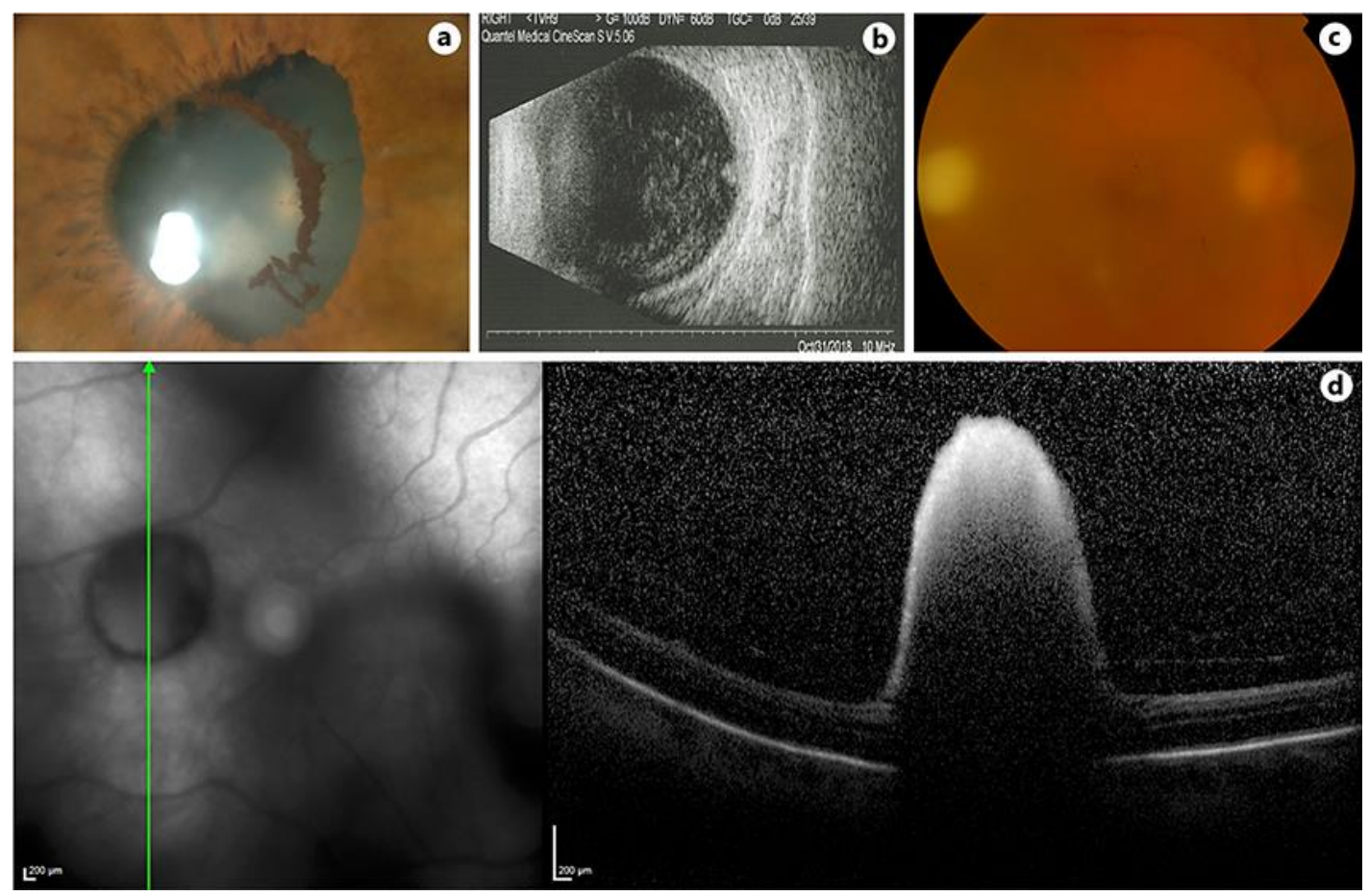

Fig. 1. Preoperative clinical presentation. a Color photographs of the anterior segment of the RE; posterior synechiae and pigment deposits on anterior crystalloid are evident. b Ten-MHz B-scan ocular ultrasound of the RE clearly documents retinal lesion and vitreous flare. c Color fundus photography of the posterior pole. $\mathbf{d}$ Horizontal OCT scan through the large infective focus shows a well-defined, hyper-reflective retinal lesion protruding into the vitreous cavity. 


\section{Case Reports in Ophthalmology}

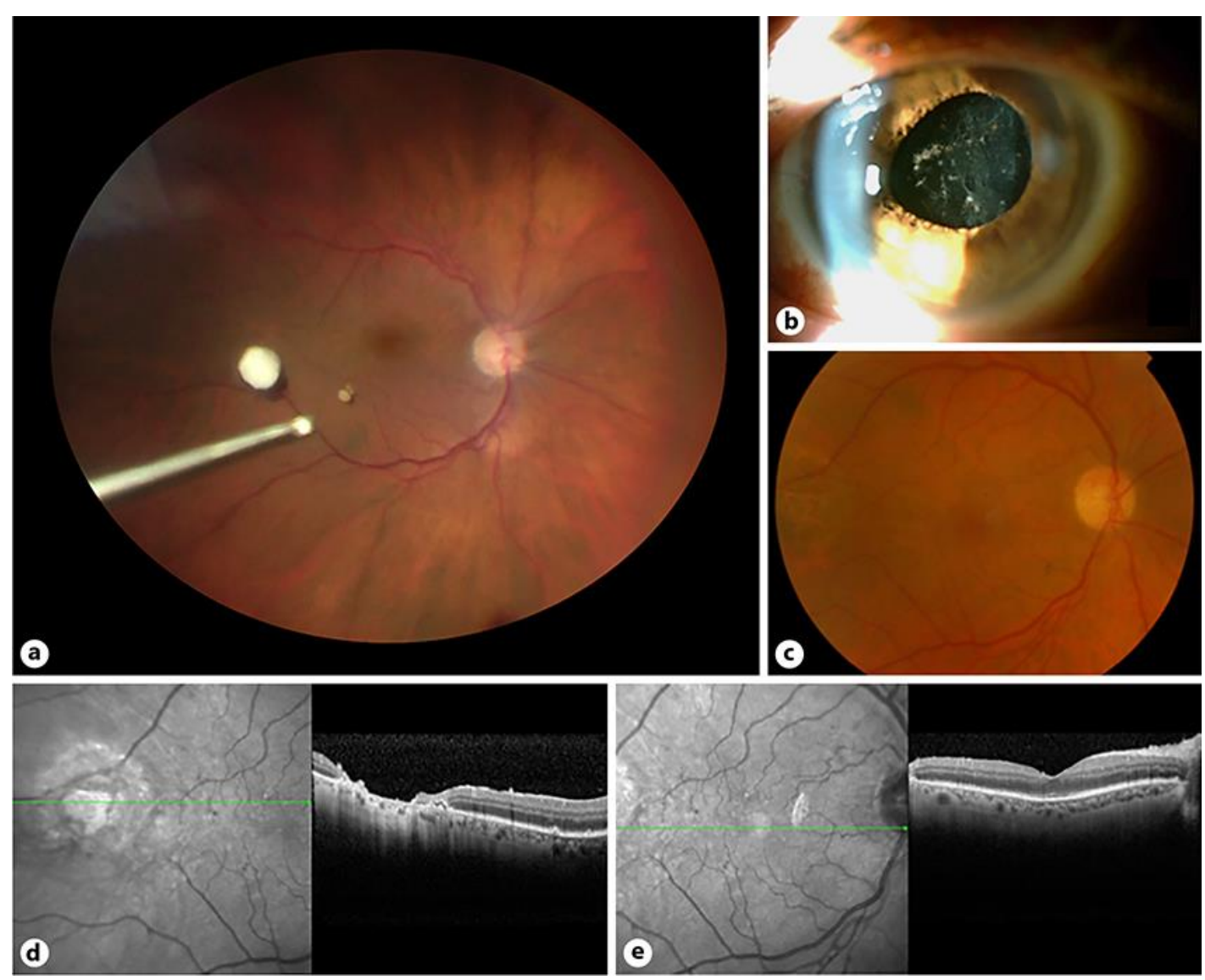

Fig. 2. a Intraoperative fundus photography: posterior pole and mid-periphery of the RE. Fungal infiltrate is visible temporally to the macula, and a smaller infiltrate can be appreciated inferotemporal to the macula. b-e Postoperative status at the 1-month follow-up. Postoperative color photographs of the anterior segment (b) and fundus (c) show a clear and quiet anterior segment and absence of retinal lesions. OCT scans reveal focal atrophy in the area where the larger infective focus was located (d) and the presence of an epiretinal membrane that does not alter the retinal thickness and profile of the macula (e). 